\title{
Conflict Detection and Resolution System Architecture for Unmanned Aerial Vehicles in Civil Airspace
}

\author{
Yazdi I. Jenie* Erik-Jan van Kampen ${ }^{\dagger}$ Joost Ellerbroek ${ }^{\ddagger}$ Jacco M. Hoekstra $^{\S}$ \\ Control and Simulation Section, Faculty of Aerospace Engineering, Delft University of Technology
}

\begin{abstract}
A novel architecture for a general Unmanned Aerial Vehicle (UAV) Conflict Detection and Resolution (CD\&R) system, in the context of their integration into the civilian airspace, is proposed in this paper. The architecture consists of layers of safety approaches, each representing a combination of different methods for surveillance, coordination, action, and decision. These are collected from a survey of various CD\&R methods existing in both manned and unmanned domains. The combination process shows that some approaches are suitable, while others are not, for the context of UAV integration. The suitable approaches are then arranged using the 'defense in depth' concept, where each layer filters a specific type of intruder. The final arrangement is set as the proposed architecture, and implemented in an assumed UAV operation within the civil airspace. System and infrastructure considerations are discussed for several examples of prospective types of operation in the airspace.
\end{abstract}

\section{Introduction}

Prospective use of an Unmanned Aerial Vehicle (UAV) for civilian domain has motivated many parties to fly their own UAVs. The increasing number of these flights in civil airspace becomes a problem, since regulations are not matured yet. Airspace authorities currently attempt to limit civil-UAVs operation within a secluded area, a policy opposed by many UAV enthusiasts. One of the examples is the attempt to run a delivery service of goods using UAVs, by a large retail company ${ }^{1 \mathrm{a}}$. Airspace authorities seem to be reluctant to allow such service and take a long time to construct an accommodating regulation. There is also an example of a UAV being used for reconnaissance of an area that has been devastated by a flood ${ }^{\mathrm{b}}$. This UAV, however, has only been flown for a short time before being grounded by the local disaster management authority. These cases demonstrate the uneasiness from both the authorities and the public regarding UAV operations; they are still considered unsafe, strange, and should only be operated in a secluded airspace. However, at the same time, people are beginning to acknowledge and enjoy their usefulness in the nonsecluded airspace, including, for instance, the use of UAVs for journalism and recreational purposes ${ }^{\mathrm{c}}$.

One possible reason of the uneasiness might be the concern about safety, especially when a UAV is sighted flying in a civil airspace with no operator to be seen. The public still has a big doubt about UAVs operating beyond the line of sight of an operator, fearing that the vehicle is uncontrolled and might hit a person, building, or even aircraft flying nearby. These fears are also reflected by some local authorities with their regulations that limit UAV operations to within the line of sight and in a secluded area. ${ }^{2}$ In short, there is a big doubt about the general Conflict Detection and Resolution (CD\&R) system, especially in autonomous mode, to support UAVs integration into civil airspace.

Researchers have been working on various approaches for the autonomous CD\&R system, which includes both hardware (sensors, computers, actuators) and software (algorithms, strategies). Although these researches show many promising results, the huge variation of approaches leads to a confusion on the integration management. Relying on only one choice of approach for the CD\&R system to resolve every conflict situation will make the UAV vulnerable, since it would only have one safety net. On the other hand, using

*PhD Student, Faculty of Aerospace Engineering, Delft University of Technology, 2629HS, Delft, The Netherlands

${ }^{\dagger}$ Assistant Professor, Faculty of Aerospace Engineering, Delft University of Technology, 2629HS, Delft, The Netherlands

${ }^{\ddagger}$ Assistant Professor, Faculty of Aerospace Engineering, Delft University of Technology, 2629HS, Delft, The Netherlands

$\S$ Professor, Faculty of Aerospace Engineering, Delft University of Technology, 2629HS, Delft, The Netherlands 
several redundant approaches is not yet practical, since they do not exactly complement each other as one general architecture for operation in the civilian airspace.

In comparison, manned-flight manages to establish a multi layered CD\&R system, applying the concept of 'defense in depth'. This architecture consists of several complementary approaches, which are commonly presented as the layers of safety ${ }^{3}$ shown in Figure 1. This architecture was not formed instantly, but rather, it was built and iterated throughout history. Most of its components exist as the result of evaluations on accidents. $^{4}$

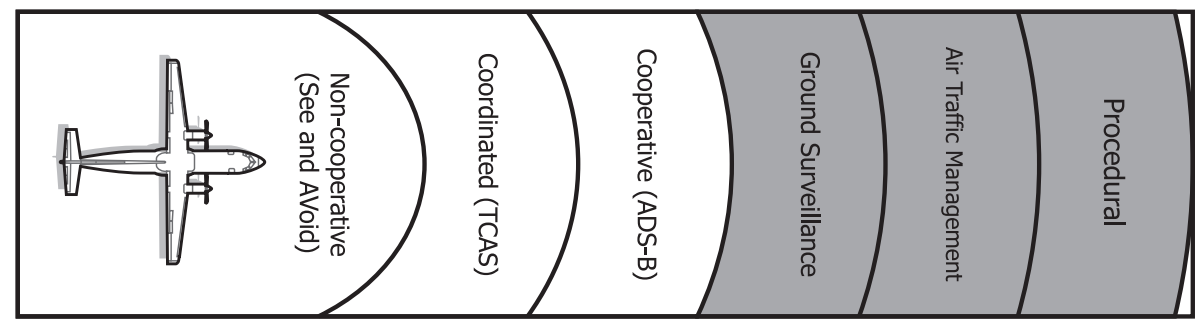

Figure 1. Layers of Safety from Manned-Flight, adapted from Ref.3

This paper proposes a novel architecture for a general UAV CD\&R system in the context of their integration into the civilian airspace. The architecture aims to arrange and manage various possible approaches in a complementary way, using the concept of defense in depth. Each approaches is viewed as a combinations of the chosen method in (1)surveillance, (2)coordination, (3)action, and (4)decision. The architecture is designed to be practical enough to be used by the authorities and operators, and to be a general guideline for developers. The last objective is to be a supplement in growing the mutual understanding among developers, operators, authorities, and public for integration of UAVs into the civil airspace.

The research in this paper is presented as follows. After the introduction, Section 2 will elaborate on the existing methods for UAV CD\&R systems, which are divided into four groups, i.e. based on surveillance, coordination, action, and decision. Those methods are then combined as approaches of CD\&R in the following Section 3. These are the components in building the architecture using the concept of defense in depth. Section 3 also elaborates on why some of the existing approaches are not suitable in the context of UAV integration in the airspace. Section 4 presents an implementation discussion of few examples of prospective UAV missions, ${ }^{5}$ including how the architecture affects the requirements for UAV. Section 5 wraps the entire research in several conclusions and suggestions for future works.

\section{CD\&R Methods and Approaches}

To give a general preview of the current available approaches for the UAV CD\&R system, a literature review is conducted and elaborated in this section. The variation of approaches can be viewed as the different combination of choices for their method of surveillance, coordination, action, and decision. For example, the manned-flight approach for their Traffic Warning and Collision Avoidance System (TCAS) is a combination of a separated independent surveillance, explicit coordination, a deconflict action, and manual decision. The following subsections explains the choice of methods a UAV has to build its CD\&R system based on the literature study. The choices of the four groups of methods are presented in Figure 2, in which they are arranged as a separated layer of safety.

\section{A. Methods for Airspace Surveillances}

Airspace surveillance is the first step in the CD\&R process, in which every possible conflicting object around the ownship should be detected. There are three types of surveillance that are currently used; the centralized-

\footnotetext{
a Gross, D., Amazon's Drone delivery: How would it work?" 2013, accessed September 2014, http://edition.cnn.com/2013/ 12/02/tech/innovation/amazon-drones-questions/

bAckerman, E., "[Updated] UAV Provides Colorado Flooding Assistance Until FEMA Freaks Out, 2013, accessed September 2014, http://spectrum.ieee.org/automaton/robotics/aerial-robots/falcon-uav-provides-colorado-flooding-assistance-until-femafreaks-out

cCorcoran, M. "Drone journalism take off", 2012, accessed September 2014, http://www.abc.net.au/news/2012-02-21/dronejournalism-takes-off/3840616
} 
dependent, separated-dependent, and independent surveillance, shown as the top set of layers in Figure 2.

A centralized-dependent-surveillance system obtains data from a common station, commonly on ground, and is available even before the flight is conducted. In manned-flight, this part is included in the first three layers, i.e., the Procedural, the Air Traffic Management, and the Ground Surveillance. The dominance in half of the layers shows the dependency of manned-flight on this surveillance. An aircraft can retrieve data about the traffic in an area, accompanied with the data about terrains and weather, from common ground centers like the Air Traffic Control (ATC) or the Aviation Weather Center (AWC). UAV operators are most likely to employ these data to plan each flight and avoid any unnecessary conflicts. An example of this practice is demonstrated in Ref. 6 and Ref. 7.

A distributed-dependent-surveillance system obtains data from the traffic itself. This surveillance method, therefore, requires cooperation from every vehicle to broadcast their flight data among each other. In manned-flight, this practice is conducted in two layers in Figure 1 which are the Automatic Dependent Surveillance Broadcast (ADS-B) ${ }^{8}$ System, and the Traffic Warning and Collision Avoid-

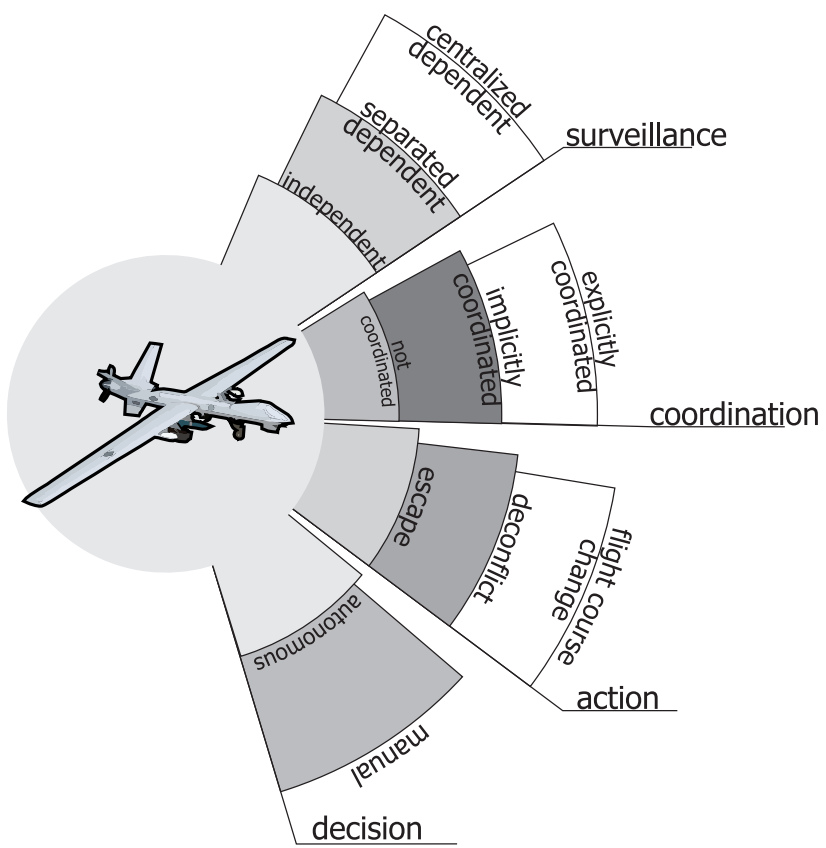

Figure 2. UAV CD\&R system: the methods of surveillance, coordination, action, and decision ance System (TCAS). Applications to UAVs have been made for both of those systems, for example as presented in Ref. 9 and Ref. 10, assuming the availability of such systems for small vehicles. In this research, vehicles that share their data using this surveillance are referred to as cooperative-vehicles.

The third method of surveillance is by obtaining airspace data independently using the on-board sensor system. In manned-flight, this independent surveillance is only the pilot eyes, used in the 'see and avoid' layer in Figure 1. While in manned-flight this type of surveillance seems to be the primordial system for avoidance, in the UAV domain it dominates in most of the researches. On-board sensors are the most popular way to provide surveillance for UAVs. This type of surveillances has been assessed, including for cameras (visual light and infra-red) ${ }^{11}$ acoustics sensors, ${ }^{12}$ and even miniaturized versions of active-sensors like laser-range-finder ${ }^{13}$ and RADAR. ${ }^{14}$

Those three types of surveillance methods are arranged in the first set of layers shown in Figure 2. The centralized-dependent-surveillance is placed as the outermost layer, since it is available even before a flight is conducted. In fact, for UAVs, centralized surveillances might only be suitable before flight, due to two reasons. Firstly, UAVs are most likely to be manufactured with a light non-metal material that is hard to detect by a ground station. Secondly, even if it can be detected, the prospective UAVs ${ }^{5}$ are commonly designed to exploit the airspace without being limited by the predefined airways used by mannedflight. Therefore, most of the time the UAV will fly in an area that is not covered by an ATC or any other common ground station. The separated-dependent-surveillance follows as the middle layer, as it is available in a range shorter than the centralize-dependent system, but generally have much longer range than independent-surveillances. The surveillance is available only when the vehicle is close enough to others that cooperatively share their flight data. The innermost layer, therefore, is the independent surveillance system.

\section{B. Methods for the Avoidance Coordination}

In order to simplify the calculation for resolution, many researches assume some level of coordination between the vehicles. This research differentiates the levels into three methods of avoidance, i.e., explicitlycoordinated, implicitly-coordinated, and uncoordinated. Avoidance is said to be explicitly coordinated if two or more specific resolutions are produced for each conflicting vehicle, from a single centralized calculation. Therefore, an explicit communication for confirmation is required among the involved vehicles. An example 
for this type of coordination can be observed in the work by an ATC or the TCAS, which gives a pair of aircraft a specific advisory to avoid conflict. There are also many works in UAV avoidance that use the method, including the next generation of TCAS for UAV, the ACAS Xu. ${ }^{4}$ In this research, a resolution calculation that is produced by the same reciprocative algorithm, even though it is conducted separately in each vehicle, is also included as an explicit coordination, such as the 'free-flight' concept presented in Ref. 15 and 16. Furthermore, collision avoidance algorithms that are intended for a multiple UAV operation, such as in Ref. 17, are also included in the explicit coordination.

An avoidance maneuver is said to be implicitly-coordinated if the involved vehicles follow a common set of rules or strategies in their avoidance. Hence, instead of explicit communication, each vehicle assumed that the other will act according to the rules that can actually simplify the resolution calculation. Being partially limited by the rules, the vehicles will still have some level of freedom in their avoidance maneuver. An example of this type of coordination in manned-flight is the right-of-way rules, which are used in the 'see and avoid' layer (see Figure 1). This rule distributes the right-of-way to the conflicting vehicle that results in, for example, some aircraft maintain their path while others conducting avoidance in a way the pilot sees fit. ${ }^{18}$ The work in Ref. 19 and Ref. 20 presents an example of this type of coordination. Furthermore, any conflict resolution methods that are under the assumption that the counterpart does not maneuver, are also included in this research as an implicitly-coordinated avoidance.

When the avoidance is uncoordinated, each involved vehicle has their own preferences for resolution based on the conflict situation, without limitation from any rules. The ownship in this case assumed that the obstacle is rogue and may conduct unexpected maneuvers. This actually makes the resolution calculation difficult since it has to take into account every possible vehicle movement and collision risks from the conflict situation. Several researchers use a predefined maneuver, commonly at the vehicle highest performance, for an aggressive sure-escape that avoids a large portion of the risk at once.

The time to calculate and produce a resolution is used as the base for the arrangement of methods into the second group of layers shown in Figure 2. The outermost layer is the explicit coordination that requires longer time, hence longer distance, especially for the confirmation process between vehicles, or from vehicles to a common ground station. The implicit coordination follows with a shorter time to process, since it can skip the confirmation part. The uncoordinated avoidance, on the other hand, can be both processed faster and slower than the other two. The process becomes fast if a vehicle uses a simple predefined automatic maneuver, or even a manual maneuver, to produce the resolution. Slow process, however, can occur if the calculation involves series of predictions taking account of every possible risk, and/or using only limited information about the dynamic of the counterpart. The fast processed calculation, however, might suit more for the limited computational power a UAV has. Therefore, the uncoordinated layer is placed on the innermost layer, without neglecting the fact that it can also be placed at the outermost layer.

\section{Methods for the Avoidance Actions}

As airborne vehicles, UAVs are able to perform many kinds of avoidance actions in the 3-Dimensional space. This research differentiates among three types of action, adopting the differentiation proposed in Ref.21, which includes the flight-course change, deconflict, and escape maneuver. A flight-course-change is the long-range action that majorly changes the initial course, in the attempt to avoid possible traffic. The flight-planning, as well as the approval from the ATC in the procedural layer (see Figure 1) of manned-flight, is included in this method of action. An example in the UAV domain can be found in Ref. 6 and Ref. 22, which presents deterministic flight planning.

Deconflict is a medium range action that changes a small part of the flight path, aiming to keep any deviation as small as possible. This action accommodates optimization in the avoidance maneuver. In the ACAS X, ${ }^{4}$ the calculation produces a deconflict type of action using a series of dynamic programming algorithms to find the most effective and safe maneuver scenario for a pair of aircraft. In this research, deconflict is assumed to be comparable with the strategic avoidance term in manned-flight. Many research commonly focuses on deconflict to produce an optimal avoidance using, for example, a cost or objective function.

The last approach is to escape any potential collision all together and conduct a maneuver solely to bring the vehicle to safety. The escape action should be aggressive, conducted as fast as possible, and driven by the maximum performance limit of the vehicle. While the other two methods bring the vehicle back to its mission in the end, this action has an option to abort the original mission. In manned-flight, this type of avoidance is similar to the tactical avoidance maneuver in the 'see and avoid' layer, in the way that 
they ignore any optimization, and focus only on the safety factor. The way the current TCAS works that follows a Resolution Advisory without any optimization process, is also included as an escape maneuver. In UAV domain, several examples use this action type, including using a maximum turning rate to escape all obstacles at once, or immediate descent whenever the sensor detects a dangerous vehicle nearby.

The arrangement of this group, as shown in Figure 2, follows the work presented in Ref. 21, which is based on the portion of the flight path changed, and therefore the distance required to conduct an action. The flight-course change action placed as the outermost layer due to the large portion of flight path it changes. A deconflict action follows as the middle layer since it changes a smaller part of the flight path, but still requires some distance to accommodate the optimization process. Escape is placed as the innermost layer as it is most likely to be applied as the last resort of avoidance that neglects the initial flight course all together.

\section{Methods for the Avoidance Decision}

Based on how the avoidance decision is made, UAVs can conduct a maneuver either manually or autonomously. In this research, the methods are differentiated more on the source of the final decision, and not on the calculation process. For instance, if a vehicle calculates a conflict situation on-board, but then send the results to the ground operator to decide, it is still counted as manual avoidance.

Manual avoidance is preferable by most of the current regulations, which require a UAV to always be within the line of sight of the ground operator. ${ }^{2}$ Beyond the line of sight, however, the effectiveness of manual avoidance is reduced greatly, as the situational awareness of the ground operator becomes low. In this case, the role of the operator should be changed to flight manager, rather than pilot. The decision for conflict avoidance, hence, should be given to the autonomous CD\&R system on-board.

The autonomous decision making process can consider many parameters at once in order to choose the best maneuver from a set of options, commonly for a short period with limited nuisance to the operator. Currently, this type of avoidance decision is not applicable in a commercial manned-flight due to safety reasons, even though many researches have been conducted in the area. For UAVs, on the other hand, autonomous avoidancve ability is the focus of most research, since it defines the intelligence as an aerial robot.

The arrangement in this group of methods is as shown in the fourth set of layers in Figure 2. The manual approach is placed as the outer layer that acts as the first means of avoidance, which is available in the first phase of the flight. The autonomous decision, therefore, is placed in the inner layer, since it is most likely to be use beyond the line of sight where manual decision is difficult to make. In some situations, however, the manual decision might be preferable for the last layer before collision. For example, in cases when there is a known failure on the vehicle on-board system, or for evasive maneuvers after a light collision. Manual avoidance might even become a requirement for the last line of defense, considering that the operator should have the most final decision to ensure safety and overwrite any other avoidance. Therefore, there is no actual order for these two methods of decision.

\section{Building the Architecture of Conflict Detection and Resolution}

The architecture proposed in this paper is built by arranging the possible approaches, which are derived from the combining the methods. Using direct combination, there are 54 possible approaches ( 3 methods of surveillance $\times 3$ methods of coordination $\times 3$ methods of action $\times 2$ methods of decision). Several of the combinations, however, will be illogical or unfeasible; not all of the 54 approaches can be used in the architecture. Instead of the direct combination, the methods are combined one-by-one. In every steps, each combination is tested whether it is suitable for the condition when the UAV is integrated into the civil airspace. This process is shown in Figure 3, and is explained thoroughly in the following subsections. These resulting approaches are then arranged as the UAV layers of safety, following the order in each set. In the next section, several examples are given for a few types of prospective UAV operations implementing the architecture.

\section{A. Combination of Methods and Eliminations}

Figure 3 shows the whole combination process. Combinations that 'survive' until the end are the appropriate approaches for applications on UAVs, especially in the context of integration into the airspace system. The combination process adds each method in one group with each method in another group one-by-one, starting 

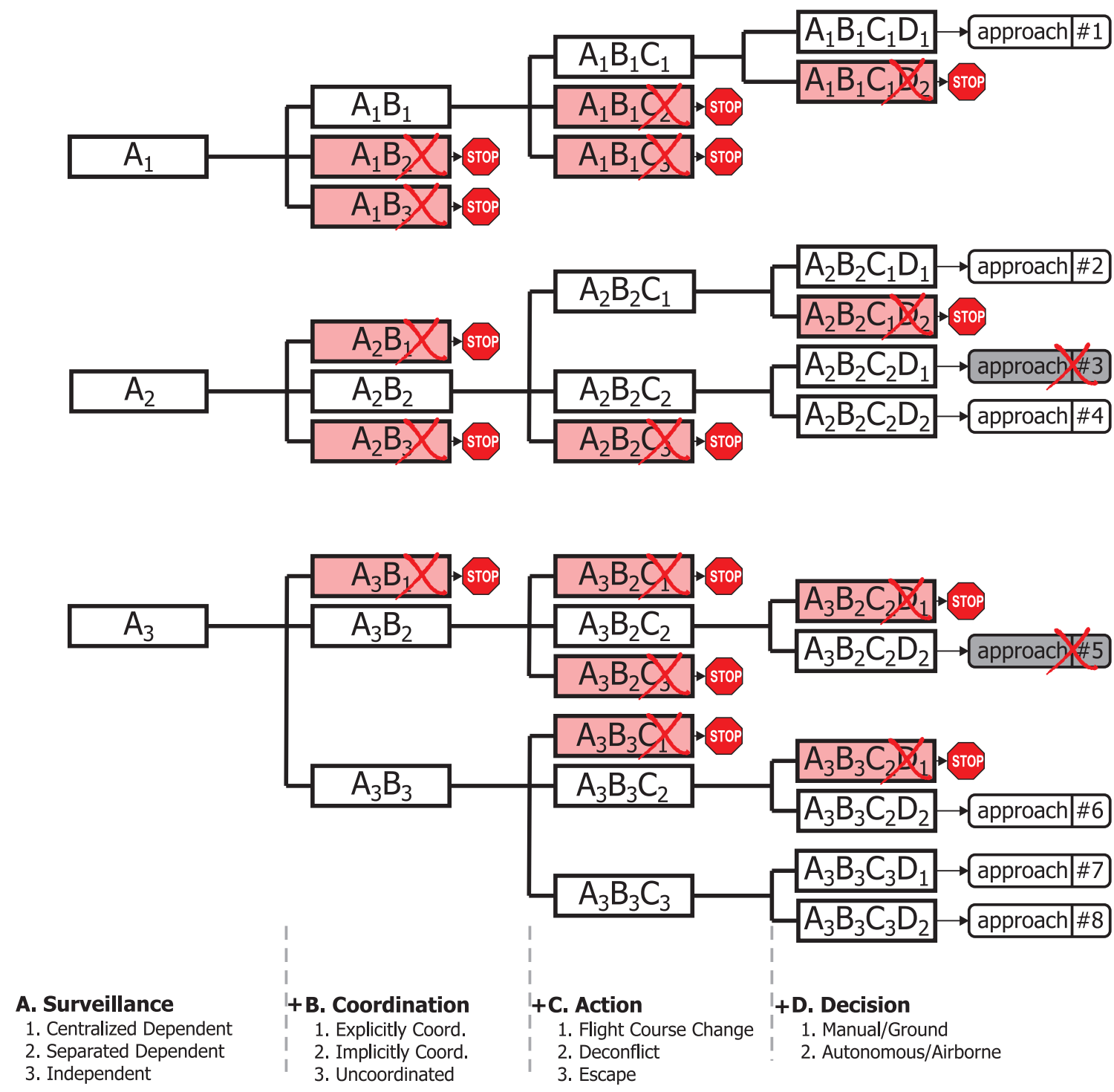

Figure 3. Combination and elimination process of four group of methods

from the methods in surveillance and ending with the avoidance decision. Some combinations, however, are discontinued due to their incompatibility with UAV applications and with the context of airspace integration. This elimination processes is explained more in the following paragraphs.

\section{Combination of the Methods for Surveillance and Coordination}

As shown in Figure 3, not all combinations of surveillance and coordination methods are accepted. There are four combinations that are eliminated due to the following reasons. The centralized-dependent-surveillance cannot support an implicitly coordinated $\left(A_{1} B_{2}\right)$ or an uncoordinated maneuver $\left(A_{1} B_{3}\right)$. These two types of coordination are most likely to be conducted when airborne, while the centralized surveillance for UAVs is only applied before the flight is conducted, as explained in section IIA. Using separated-dependent surveillance for an explicit coordination $\left(A_{2} B_{1}\right)$ is also rendered unfeasible, since it would require each UAV to have the same system, like the TCAS in the manned flight. Assuming that when it is integrated, UAVs types will vary wildly in size and mission, it is actually impractical to enforce all of them to carry the same system with the same algorithm for resolution. The surveillance combination with the uncoordinated maneuvers $\left(A_{2} B_{3}\right)$, furthermore, is also discontinued. The reason is that if every vehicle cooperatively shares their flight-data, it will not make sense if they act without any coordination. Lastly, the independent surveillance naturally 
cannot be used to support an explicit coordination $\left(A_{3} B_{1}\right)$, since there is no communication between vehicles.

\section{Combination with the Methods for Action}

The combination process is then continued with the methods of action, as shown by the third column of Figure 3. There are twelve possible combinations that can result from this process, of which six are discontinued. Similar to the coordination methods, the second and third row of combinations $\left(A_{1} B_{1} C_{2}\right.$ and $\left.A_{1} B_{1} C_{3}\right)$ are eliminated since the centralized-dependent-surveillance can neither support the action of deconflict or escape, due to its preflight/ground only applicability.

The separated dependent surveillance could actually support all three types of action. The combination with the action of escape $\left(A_{2} B_{2} C_{3}\right)$, however, is unnecessary, and hence discontinued, considering the range covered by the surveillance and the cooperative nature of the involved aircraft. Flight-course-change action, on the other hand, can be implicitly coordinated with the support of the separated-dependent-surveillance. For example, if a UAV is about to fly trough an area that already has traffic within, then, by using a simple 'first-come-first-serve' rule, the UAV might need to conduct a local recalculation of its path, and avoids without bothering the others. The same situation can also be conducted using the deconflict action, in a shorter range. Alternate rules can be implemented in this case, for example, using the VFR right-of-way rules that distribute the right of way to the vehicles according to the conflict geometry, demonstrated in Ref. $^{20}$

Combining the independent-surveillance with an implicitly coordinated avoidance can only be realized with a deconflict action. Independent surveillance has a relatively short range of detection such that it does not make sense to ask another UAV to change a major part of its course using the action of flightcourse-change, to follow some implicit coordination $\left(A_{3} B_{2} C_{1}\right)$. The same reason is used to eliminate the combination of uncoordinated flight course change action with the independent surveillance $\left(A_{3} B_{3} C_{1}\right)$. An implicit coordination with deconflict action, in contrast, is still accepted, since it would only enforces small changes to others. The escape action is also incompatible with an implicit coordination $\left(A_{3} B_{2} C_{3}\right)$, because the ownship cannot always expect the counterpart to do an aggressive maneuver.

The support of an independent-surveillance for an uncoordinated avoidance can be realized using either the deconflict or the escape action. The former is a popular method use in many studies that aim to conduct the most efficient UAV uncoordinated avoidance, based on on-board sensor. The latter is usually the solution for fast avoidance to safety whenever there is only limited information available on the conflict.

\section{Combination with the Methods of Decision}

The fourth and final column of Figure 3 represents the combination process with the methods of decision, whether it is made manual or autonomous. From the remaining six, twelve more methods can be produced in this level. The action of flight-course change is most likely to be conducted manually, considering the distance and the major part of the course that will be changed. Therefore, two of the combinations $\left(A_{1} B_{1} C_{1} D_{2}\right.$ and $\left.A_{2} B_{2} C_{1} D_{2}\right)$ are eliminated. The decision to deconflict, on the other hand, can be both manually or autonomously. If it is supported by an independent-surveillance $\left(A_{3} B_{2} C_{2} D_{1}\right)$, the situational awareness at ground is not sufficient, and hence eliminated. Moreover, even if there is an independent-surveillance that is accurate enough, the detection range is already too close for the process of data exchange to the ground operator. Therefore, the manual avoidance is eliminated $\left(A_{3} B_{3} C_{2} D_{1}\right)$ in the independent surveillance group.

The last combination in the fourth column of Figure 3, however, actually presents a manual escape action that is supported by an independent surveillance. This is a special case of escape in a situation where the ground operator realizes that all other measures cannot ensure safety, for example, if there is a known failure on the avoidance computational system.

\section{Further Elimination}

One of the disadvantages of using 'defense in depth' concept is that if the depth is too deep, then the resulting system becomes too complex and confusing. Even though each of the eight resulted approaches is possible and suitable to be implemented in a UAV, there are some overlapping functions that make the whole architecture become too complex. For instance, Approach \#3 and \#4 overlap each other in their function; both of them are using separated-dependent-surveillance to conduct an implicitly-coordinated deconflict, which means that they are avoiding the same set of cooperative vehicle in a relatively same 
distance, set for a deconflict action. Then most likely if the manual (Approach \#3) avoidance is conducted, the autonomous avoidance (Approach \#4) is not needed. Instead, the next step of avoidance should skip directly to Approach \#5, avoiding obstacles that have not been avoided, the non-cooperative ones, which are detected using an independent surveillance. Hence, the third and fourth approach are merged by focusing more on the autonomous capability of a UAV.

Approach \#5 and \#6 are overlapping as well. Obstacles that already penetrate through the previous layer are non-cooperative vehicles, thus cannot be expected to follow any implied rules. There is also a chance that those obstacles are actually cooperative, but do not share their data because of some failure in the system. However, it would be unreasonable to only conduct an avoidance that takes into account the all the risk, after the distance has been significantly reduced when expecting the obstacles to obey a set of rules. Therefore, Approach \#5 which assumes the vehicles would follows some implicit coordination, is removed.

\section{B. Arrangement and Comparison}

From the series of reasoning in the previous paragraph, six approaches remain, as shown in the white boxes on the right side of Figure 3. These approaches are assumed to be appropriate for UAVs and for their integration into the airspace. The architecture of UAV CD\&R system can be built using these six approaches that are arranged in layers of safety, similar to the manned-flight's in Figure 1. Conveniently, these are already arranged, vertically from the top, according to the order specified in each group of methods (see Section II). A switch, however, needs to be made for the last two methods. A manual uncoordinated escape should be used after an autonomous one, since it serves as an emergency override, the last resort for avoidance. This is where the operator has an executive decision to escape unexpected incidents like, for example, an on-board system failure.

The final arrangement is then presented in Figure 4, along with the methods applied in each of layers. Each layers is designated with a name that represent its characteristics, to simplify further discussion. Other examples for layered safety design for a CD\&R system can also be found in Ref. 23, Ref. 21, Ref. 24, and Ref. 25 as well. The problem of integration, however, has not been thoroughly investigated in those designs. More similarity can be found in the work in Ref. 26, that presents the Jointly Optimal Conflict Avoidance (JOCA). The work starts from the approaches for conflict resolution that are used in manned-flight to set a hierarchy of constraints, which have to be satisfied as much as possible according to the specific situation.

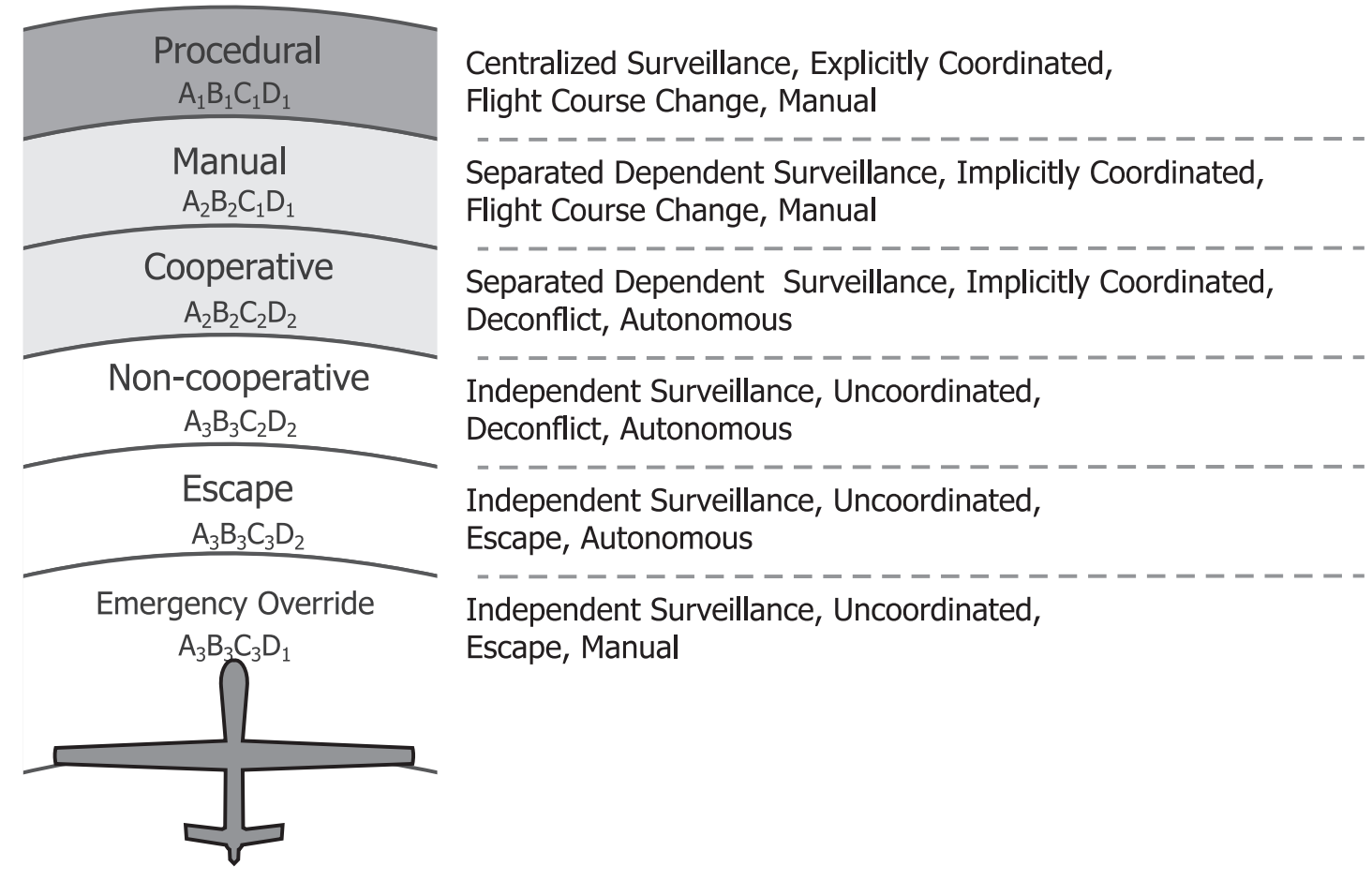

Figure 4. The novel architecture, presented as layers of safety for UAVs operation in the civil airspace 
Table 1. Existing and/or suitable combinations of methods for UAVs in civil airspace

\begin{tabular}{|c|c|c|c|c|c|}
\hline \multirow[b]{2}{*}{ Surv. } & \multicolumn{2}{|c|}{ Combination } & \multirow[b]{2}{*}{ Deci. } & \multirow[t]{2}{*}{ Designation } & \multirow[t]{2}{*}{ Examples } \\
\hline & Coor. & Acti. & & & \\
\hline$\overline{A_{1}}$ & $B_{1}$ & $C_{1}$ & $\overline{D_{1}}$ & Procedural & Ref. $27^{\dagger}$ \\
\hline$A_{1}$ & $B_{1}$ & $C_{1}$ & $D_{2}$ & - & Ref. $6 \dagger^{\dagger} 22,17 \ddagger 28$, and $29^{\dagger}$ \\
\hline$A_{1}$ & $B_{1}$ & $C_{2}$ & $D_{1}$ & - & Ref. 30 \\
\hline$A_{1}$ & $B_{1}$ & $C_{2}$ & $D_{2}$ & - & Ref. 31,10 , and 32 \\
\hline$A_{1}$ & $B_{2}$ & $C_{1}$ & $D_{2}$ & - & Ref. 33 \\
\hline$A_{1}$ & $B_{3}$ & $C_{3}$ & $D_{2}$ & - & Ref. 34 \\
\hline$A_{2}$ & $B_{1}$ & $C_{2}$ & $D_{2}$ & - & Ref. 35,36 , and 37 \\
\hline$A_{2}$ & $B_{2}$ & $C_{1}$ & $D_{1}$ & Manual & Ref. 6 \\
\hline$A_{2}$ & $B_{2}$ & $C_{2}$ & $D_{2}$ & Cooperative & Ref. 19,38 , and 20 \\
\hline$A_{2}$ & $B_{3}$ & $C_{2}$ & $D_{2}$ & - & Ref. 39 and 4 \\
\hline$A_{2}$ & $B_{3}$ & $C_{3}$ & $D_{1}$ & - & Ref. 40 \\
\hline$A_{3}$ & $B_{1}$ & $C_{1}$ & $D_{2}$ & - & Ref. $41^{\dagger}$ and $42^{\dagger}$ \\
\hline$A_{3}$ & $B_{1}$ & $C_{2}$ & $D_{2}$ & - & 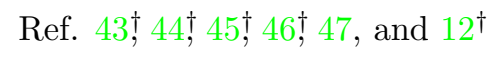 \\
\hline$A_{3}$ & $B_{1}$ & $C_{3}$ & $D_{1}$ & - & Ref. $48^{\dagger}$ \\
\hline$A_{3}$ & $B_{1}$ & $C_{3}$ & $D_{2}$ & - & Ref. $49^{\dagger}$ \\
\hline$A_{3}$ & $B_{2}$ & $C_{2}$ & $D_{2}$ & - & Ref. $50,51,13,52,53$, and 54 \\
\hline$A_{3}$ & $B_{3}$ & $C_{2}$ & $D_{2}$ & Non-cooperative & - \\
\hline$A_{3}$ & $B_{3}$ & $C_{3}$ & $D_{1}$ & Escape & Ref. 34 \\
\hline$A_{3}$ & $B_{3}$ & $C_{3}$ & $D_{2}$ & Emergency Override & - \\
\hline
\end{tabular}

$\dagger$ Indoor application, against static obstacles.

$\ddagger$ Intended for multiple UAV operation.

Due to the series of reasoning also, some existing methods for a Conflict Detection and Resolution are rendered as not suitable. Table 1 shows, from the references used, the matching and un-matching approaches. Note that the list in the table is in the order of the combination process from Figure 3. The table also omits many combinations that do not have any examples in the references used. It should be noted that some of the references are not exclusively in the UAV domain, but in manned-flight as well.

From Table 1, it can be shown that most approaches do not comply with the architecture proposed in this paper. For example, the manned-flight TCAS-like protocol $^{39,4}$ is not suitable because the assumption that UAVs will exploit the airspace with a large variety of types, which makes it difficult to force every of them to have the same algorithm for an explicit coordination. Another example is an explicitly coordinated deconflict that relies on centralized-dependent-surveillance. ${ }^{10,31,35,32}$ This approach is unpractical since it requires a ground station that is able to track UAVs all the time.

Two layers in the architecture, on the other hand, do not have any examples in the references. Many researches actually intended to propose approaches for the Non-cooperative Layer, an autonomous uncoordinated deconflict avoidance that is based on the independent-surveillance. ${ }^{50,51,13,52,53,54}$ However, it is not rendered as suitable for the layer, since most of them assume that the counterparts are non-maneuvering, hence, they are actually implicitly-coordinated. An example of a true uncoordinated avoidance, one that take into account the counterpart maneuverability, is in Ref.35. However, this example assumes a communication between the UAVs using a separated-dependent-surveillance. Moreover, examples of the Emergency Override are also hard to find, since this layer can only be relevant if the UAVs are already integrated. Instead, the table shows many researches that are still focused on indoor application and conflicts with static obstacles, reflecting the current situation of UAVs acceptance. 


\section{General Implementation}

Following the concept of defense in depth, each layer in the architecture applies a single approach to prevent a specific obstacle penetrating the next layer. Parameterization is required to define the threshold of each layer. Two parameters that have been considered are the distance between vehicles, and the time-to-collision. ${ }^{21}$ It should be noted that it is not necessary for the layers to be incremented uniformly nor should they adapt a specific shape or volume. Two layers might not have a clear line to differentiate them. The exact parameterization is not discussed further in this research, since it will depend highly on the type of UAV. An illustration of the implementation of the layers, is given in the following paragraphs.

The layers of safety are generally implemented as follows. First, before the flight is even conducted, the operator seeks approval for the flight-plan for the UAV mission. This activity is represented by the Procedural Layer. The aim is to avoid any unnecessary conflict with other traffic, static obstacles, or bad weather. This is done with a centralized surveillance such as an Air Traffic Control (ATC) station, or other network stations. The operator, hence, is responsible to give the designated flight plan back to the center. This layer filters any unnecessary conflicts from the UAV operation.

After departure, the UAV relies first on its dependent surveillance system, which, with the current technology, can be either the ADS-B, or the FLARM ${ }^{\mathrm{d}}$. The system detects other cooperative vehicles both manned and unmanned, early enough to send the updated traffic data to the ground, and conduct, manually decided, a flight course change. This is the Manual layer of the architecture. The implicit-coordination in this layer can be a simple rule like, for example, not to bother the existing traffic (first-come-first-served). This layer will avoid any cooperative obstacles that are detected early enough to select other alternatives.

In cases where the change of flight course is not preferable due to mission importance like saving fuel, the UAV can choose to avoid with a deconflict maneuver, implementing the Cooperative Layer. Another example is when, due to some failures, the cooperative counterpart is not detected soon enough for a flightcourse change. The maneuver in this layer is conducted autonomously due to the assumed lack of situational awareness of the ground operator. Implicit rules, such as an adaptation of the manned-flight Visual Flight Rules (VFR), ${ }^{18}$ can be applied to simplify the resolution. At this point, all conflicts with normal mannedaircraft are resolved.

By using independent-surveillance, the last three layers are actually substitutes to the 'see and avoid' layer in manned flight. The Non-cooperative Layer intends to avoid obstacles that are able to penetrate the previous three layers, which can be non-cooperative vehicles, or cooperative vehicles with some system failures. The uncoordinated maneuver should be used either way to take account of all the risk. The type of independent surveillance will determine the distance where the layer is applied. In this layer, every conflict with normal aircraft, manned or unmanned, is resolved.

The Escape Layer aims to avoid any remaining non-cooperative obstacles that are hard to detect within sufficient range for a deconflict maneuver. With the advancement of independent surveillance technology, however, the probability to encounter these obstacles is small. An escape should be aggressive using the maximum ability of the vehicle to avoid collision. There will only be a very short distance left for the calculation process, so a predefined sure-escape maneuver is preferable. Therefore, the vehicle's maneuverability should be the deciding factor in implementing the layer. The work of Ref. 21, for example, sets the time of 1.5 second-to-collision to start escaping.

Due to various unexpected situations, penetrations through all the five previous layers are still possible. For example, there might be a failure in the autonomous CD\&R system, or an unpredicted acceleration from the counterpart. In these situations, the operator on the ground can take over and conduct an avoidance sooner, compared to the autonomous processes. This also applies in situations where a collision has occurred, but only partially damages the own-ship. The operator have the emergency override to prevent any further damage, including, for example, preventing the crippled UAV to fall into a populated area.

\section{CD\&R system consideration in Examples}

The implementation of the defined layers of safety in Figure 4 depends on the mission of the UAV. For some missions, some layers might become unnecessary, while others become important. Some of the layers can impose a requirements to the UAV CD\&R system. This section presents a few examples of the implementation of the architecture for a few prospective UAV operation, given in Table 2, based on the work

\footnotetext{
d_. "FLARM: What Is It", -, accessed September 2014, http://www.gliderpilot.org/Flarm-WhatIsIt
} 
Ref. 5 with a few additions. This research limits itself to these categories of UAVs, without ruling out others that might already, or soon will, exist.

Table 2. Prospective UAVs operation in the civil airspace, partly adapted from Ref. 5

\begin{tabular}{lccccc}
\hline Mission & $\begin{array}{c}\text { Operational } \\
\text { Weight }\end{array}$ & $\begin{array}{c}\text { Cruising } \\
\text { Speed }\end{array}$ & $\begin{array}{c}\text { Cruising } \\
\text { Altitude }\end{array}$ & $\begin{array}{c}\text { Maximum } \\
\text { Range }\end{array}$ & Endurance \\
\hline \hline Telecommunication Airship $^{5,55,56}$ & $20 \mathrm{ton}$ & $\mathrm{n} / \mathrm{a}$ & $20 \mathrm{~km}$ & $\mathrm{n} / \mathrm{a}$ & 1 year \\
High-Altitude Imagery $^{5,57}$ & $800 \mathrm{~kg}$ & $96 \mathrm{~km} / \mathrm{h}$ & $18 \mathrm{~km}$ & $3 \mathrm{~km}$ & 9 months \\
Border Patrol $^{5,58}$ & $25 \mathrm{~kg}$ & $50 \mathrm{~km} / \mathrm{h}$ & $<6 \mathrm{~km}$ & $25 \mathrm{~km}$ & $\mathrm{n} / \mathrm{a}$ \\
Maritime Surveillance $^{5,59}$ & $20 \mathrm{~kg}$ & $133 \mathrm{~km} / \mathrm{h}$ & $<6 \mathrm{~km}$ & $500 \mathrm{~km}$ & $5 \mathrm{hr}$ \\
Environmental Sensing $^{5,60}$ & $25 \mathrm{~kg}$ & $50 \mathrm{~km} / \mathrm{h}$ & $<6 \mathrm{~km}$ & $25 \mathrm{~km}$ & $40 \mathrm{~min}$. \\
Media and Traffic Reporting $^{5,61}$ & $10 \mathrm{~kg}$ & $60 \mathrm{~km} / \mathrm{h}$ & $<1.5 \mathrm{~km}$ & $25 \mathrm{~km}$ & $40 \mathrm{~min}$. \\
Law Enforcement $^{5,62}$ & $25 \mathrm{~kg}$ & $10 \mathrm{~km} / \mathrm{h}$ & $<120 \mathrm{~m}$ & $\mathrm{n} / \mathrm{a}$ & $8 \mathrm{hr}$. \\
Delivery Service $^{1}$ & $<25 \mathrm{~kg}$ & $80 \mathrm{~km} / \mathrm{h}$ & $<120 \mathrm{~m}$ & $25 \mathrm{~km}$ & $30 \mathrm{~min}$. \\
\hline
\end{tabular}

The implementation is discussed under the assumption that UAVs are already integrated in the airspace system. The integration is assumed to allow UAV operation in civil airspace as detailed in Ref. 5 . The aerodrome airspace (class B, C and D), however, remains highly restricted. Class A airspace is also restricted for the main operation of any UAV, except for short periods, like climbing for a High-Altitude-Long-Endurance (HALE) UAV. Class E becomes the airspace with the highest risk since both (small) manned-aircraft, as well as UAVs, are flying in there. The prospective UAV operation in Table 2 is grouped into three types of UAVs, i.e., HALE, Rural Surveillance, and Urban Low Altitude UAVs.

\section{A. High Altitude Long Endurance (HALE) UAV}

Two UAV types and missions in Table 2 is included in the HALE -UAV category, i.e., the Stratospheric Telecommunications Airship, ${ }^{55,56}$ and High-Altitude Imagery. ${ }^{57}$ Both of them operate at altitudes over 20 $\mathrm{km}$, higher than the class A airspace where commercial aircraft commonly cruise. Both of them are most likely will have low maneuverability, considering their type, i.e. an airship and a very-high aspect-ratio fixed wing. This will induce some difficulty in applying layers that use the action of deconflict or escape. The vehicle, therefore, will rely more on flight-course-changes for avoidance.

A HALE -UAV will depart from a rural base, and directly climb to its operational altitude. This short climb phase might involve some encounters with various other UAVs and small manned-aircraft in the class E airspace, and with big commercial aircraft in the class A-airspace as well. The safest way for the operation is to fly in a secluded area away from any manned flight. In cases where it cannot be fully secluded, however, the possibility of encounters with manned-flight will require the UAV to communicate its flight-plan with an Air Traffic Control (ATC) to obtain some level of clearance. A HALE-UAV area of operation, the upper class-E airspace, on the other hand, can almost be considered as physically secluded from other UAV and manned aircraft.

For the dependent surveillance system, which in the current technology can be the ADS-B,$^{8}$ or the FLARM. The manned-flight's ADS-B is more preferable due to its longer range of detection (until 185 $\mathrm{km}$ ), compared to the $5 \mathrm{~km}$ the FLARM can offer. The long range of detection allows for more maneuver possibilities, especially for a vehicle with low maneuverability. A TCAS is also an option to be more compatible with the current manned-flight system, but will add an extra $10 \mathrm{~kg}$.

For the independent surveillance, the most popular choice currently is a visual light camera system, which has detection range up to $10 \mathrm{~km}$. However, especially for a large airship, a system with further detection range like an airborne RADAR is also a choice. The system will be suitable for detecting a metal manned-flight, the most important vehicle to be avoided, with a detection range of $15 \mathrm{~km}$. The weight of such system, however, reaches $25 \mathrm{~kg}$, which can significantly reduce the payload capacity. Current research that is working on airborne RADAR miniaturization ${ }^{14}$ might open other possibility in the near future. 


\section{B. Rural Surveillance UAV}

The Border Patrol, ${ }^{58}$ Maritime Surveillance, ${ }^{59}$ and Environmental Sensing UAV ${ }^{60}$ are included in Tactical Surveillance UAVs. Those three operate in the class E airspace under 18,000 ft, where there is a possibility to encounter other similar types of UAVs. Weighing around $25 \mathrm{~kg}$, this type cannot carry a heavy surveillance system such as the manned-flight version of the ADS-B. ${ }^{8}$ Instead, the FLARM, that is under 500 grams, is more preferable. Different from the HALE, most of these UAVs have high maneuverability, thus can rely more on deconflict maneuver for avoidance. There will also be more variation in aircraft type, which range from a hand launched fixed-wing to a vertical take-off rotary wing. Considering its medium endurance and area of operation, the UAV will depart from a rural base and operates away from populated area.

Similar to the HALE, this type of UAV needs to communicate its flight plan with an ATC as the centralized dependent surveillance. The clearance should consider small manned aircraft, and any registered UAV flying in the area. The clearance, however, might not be mandatory, since these UAVs are also meant for event-based or emergency operation, for example, a Maritime Surveillance UAV flight to survey a tanker accident. Therefore, the avoidance should be relying more on the other two surveillance systems. Manual decision will have a small role, because of the short range of detection the FLARM can provide for the Manual Layer. Therefore, in most of its operation the UAV will implement directly the third layer, and avoid obstacle using an implicitly coordinated deconflict maneuver. Rules such as the VFR are used for the avoidance, especially in the enounter with other UAVs. In emergency situations such as a search and rescue operation, however, the UAV might have the highest priority among others, and thus override the VFR.

A light weight camera system can be used for its independent surveillance. The UAV developer can also consider combining the surveillance system with the camera the UAV uses for its main task. The distance to start the Non-cooperative Layer, however, needs to be determined based on, for example, the risk imposed by a non-cooperative counterparts.

\section{Urban Low Altitude UAV}

The Urban Low Altitude UAV describes three of the UAVs in Table 2, including the Media and Traffic Reporting, ${ }^{61}$ Delivery Service, ${ }^{1}$ and Tactical Law Enforcement UAVs. ${ }^{62}$ These UAVs operate in low altitude below $1.5 \mathrm{~km}$, and mostly above urban areas like cities, inside the class-G airspace. The UAVs are commonly electric-powered rotary wings, which are able to hover and perform other narrow-spaced maneuvers. This type of UAV probably has the largest number of interested companies, hoping to be able to fly it from the roof of their buildings. The flight, therefore, needs to take account of static obstacles, like towers and buildings, more than the HALE or the Rural Surveillance UAV. During its low altitude and short period of flight, it is almost impossible for it to encounter manned flight. The operation is secluded in the city area, and probably its greatest dynamic treat are birds and other UAVs. Flight into the airport aerodrome should be highly restricted.

The procedural layer implementation will require many infrastructure developments. On departure, the UAV needs to match the schedule of in its own departing point. A Delivery UAV may require some sort of a clearance or permission from the end point. Instead of an ATC, the centralized-dependent-surveillance for this layer requires a new establishment among the urban operators, and should also be accessible for the authorities and general public, i.e., by using the Internet. An on-line tracking system would be preferable. Data from this establishment should also include information about buildings and towers and other landmarks. FLARM is a definite choice for the separated dependent surveillance in the current technology. A special type of surveillance can be made for urban applications, along with their special implicit rules. For the independent surveillance, a camera system is an option, but other types of sensors are also being developed in a rapid phase that can be lighter and lower in power consumption. The detection range requirements can be short due to the high maneuverability of the UAV. Special precaution, like a parachute system, should be made in the Emergency Override layer, to avoid falling in a populated area or crashing into a building.

\section{Conclusion}

It is shown that approaches for an Unmanned Aerial Vehicle (UAV) Conflict Detection and Resolution $(C D \& R)$ system can be viewed as combinations of chosen methods of surveillance, coordination, action and decision. Some of the methods, however, are unavailable for UAV application, for example, the Air Traffic Control (ATC) station. Furthermore, several of the approaches are not suitable to support UAVs integration 
into the civil airspace, even though these approaches have been used in various researches.

A novel architecture for UAVs CD\&R system is derived by arranging appropriate approaches using the defense in depth concept. The layers represent the means of safety a UAV is required to have for its operation. It should be noted that some layers might be less significant due to operational limitations. The architecture can act as a guideline for developers to consider the design for the UAV, as well as the infrastructure. Parameterization of the layers for further definition of requirements still needs to be conducted for all UAV types and missions. This is left for the future work toward UAV integration into the civil airspace system.

\section{References}

${ }^{1}$ D'Andrea, R., "Guest Editorial Can Drones Deliver?" Automation Science and Engineering, IEEE Transactions on, Vol. 11, No. 3, July 2014, pp. 647-648, doi: 10.1109/TASE.2014.2326952.

${ }^{2}$ UK Civil Aviation Authority, CAP722, Unmanned Aircraft System Operations in UK Airspace Guidance..

${ }^{3}$ Dalamagkidis, K., Valavanis, K. P., and Piegl, L. A., "On Integrating Unmanned Aircraft Systems into the National Airspace System," International Series on Intelligent Systems, Control, and Automation: Science and Engineering, Vol. 36, Springer Science+Business Media, 1st ed., 2009, doi: 10.1007/978-94-007-2479-2.

${ }^{4}$ Kochenderfer, M. J., H. J. E. C. J. P., "Next Generation Airborne Collision Avoidance System," Lincoln Laboratory Journal, Vol. 19, No. 1, 2012, pp. 55-71, doi: 10.2747/1548-1603.48.1.24.

${ }^{5}$ DeGarmo, M. T. and Nelson, G. M., "Prospective unmanned aerial vehicle operations in the future national airspace system," AIAA 4th Aviation Technology, Integration, and Operations Forum, ATIO, AIAA, 2004, pp. 172-179, AIAA 20046243, doi:10.2514/6.2004-6243.

${ }^{6}$ Nikolos, I., Valavanis, K., Tsourveloudis, N., and Kostaras, A., "Evolutionary Algorithm Based Offline/Online Path Planner for UAV Navigation," IEEE Transactions on Systems, Man, and Cybernetics, Part B: Cybernetics, Vol. 33, No. 6, 2003, pp. 898-912, doi: 10.1109/TSMCB.2002.804370.

${ }^{7}$ Brooker, P., "Introducing unmanned aircraft systems into a high reliability ATC system," Journal of Navigation, Vol. 66, No. 5, 2013, pp. 719-735, doi: 10.1017/S0373463313000337.

${ }^{8}$ McCallie, D., Butts, J., and Mills, R., "Security analysis of the ADS-B implementation in the next generation air transportation system," International Journal of Critical Infrastructure Protection, Vol. 4, No. 2, 2011, pp. 78-87, doi: 10.1016/j.ijcip.2011.06.001.

${ }^{9}$ Zeitlin, A. b. and McLaughlin, M. b., "Safety of cooperative collision avoidance for unmanned aircraft," IEEE Aerospace and Electronic Systems Magazine, Vol. 22, No. 4, 2007, pp. 9-13, doi: 10.1109/MAES.2007.351714.

${ }^{10}$ Park, J.-W., Oh, H.-D., and Tahk, M.-J., "UAV collision avoidance based on geometric approach," 2008, doi: 10.1109/SICE.2008.4655013

${ }^{11}$ Fasano, G., Accardo, D., Moccia, A., Carbone, G., Ciniglio, U., Corraro, F., and Luongo, S., "Multi-sensor-based fully autonomous non-cooperative collision avoidance system for unmanned air vehicles," Journal of Aerospace Computing, Information and Communication, Vol. 5, No. 10, 2008, pp. 338-360, doi: 10.2514/1.35145.

${ }^{12}$ Mller, J., Ruiz, A., and Wieser, I., "Safe and sound: A robust collision avoidance layer for aerial robots based on acoustic sensors," 2014, doi: 10.1109/PLANS.2014.6851492.

${ }^{13}$ Hrabar, S. b., "Reactive obstacle avoidance for rotorcraft UAVs," 2011, doi: 10.1109/IROS.2011.6094560.

${ }^{14}$ Moses, A., Rutherford, M., Kontitsis, M., and Valavanis, K., "UAV-borne X-band radar for collision avoidance," Robotica, Vol. 32, No. 1, 2014, pp. 97-114, doi: 10.1017/S0263574713000659.

${ }^{15}$ Hoekstra, J. M., "Designing for Safety: the Free Flight Air Traffic Management concept," 2001.

${ }^{16}$ Hoekstra, J., van Gent, R., and Ruigrok, R., "Designing for safety: the free flight air traffic management concept," Reliability Engineering 83 System Safety, Vol. 75, No. 2, 2002, pp. 215 - 232, doi: 10.1016/S0951-8320(01)00096-5.

${ }^{17}$ Maza, I., Caballero, F., Capitn, J., Martnez-De-Dios, J., and Ollero, A. b., "Experimental results in multi-UAV coordination for disaster management and civil security applications," Journal of Intelligent and Robotic Systems: Theory and Applications, Vol. 61, No. 1-4, 2011, pp. 563-585, cited By (since 1996)20.

${ }^{18}$ Federal Aviation Administration, Federal Aviation Regulations (FAR) Chapter I, subchapter F Air Traffic and General Operating Rules, Section 91.113 Right-of-way rules: Except water operations.

${ }^{19}$ Le Tallec, C., "VFR general aviation aircraft and UAV flights deconfliction," Aerospace Science and Technology, Vol. 9, No. 6, 2005, pp. 495-503, doi: 10.1016/j.ast.2005.01.001.

${ }^{20}$ Jenie, Y. I., van Kampen, E., de Visser, C. C., and Chu, Q. P., "Selective Velocity Obstacle Method for Cooperative Autonomous Collision Avoidance System for UAVs," AIAA Guidance, Navigation, and Control Conference 2013, AIAA, Boston, MA, 2013, AIAA 2013-4627, doi:10.2514/6.2013-4627.

${ }^{21}$ Barfield, F., "Autonomous collision avoidance. The technical requirements," National Aerospace and Electronics Conference (NAECON), 2000, pp. 808-813, doi:10.1109/NAECON.2000.894998.

${ }^{22}$ Borrelli, F., Subramanian, D. d., Raghunathan, A., and Biegler, L., "MILP and NLP techniques for centralized trajectory planning of multiple unmanned air vehicles," 2006, cited By (since 1996)15.

${ }^{23}$ Yang, L. C. and Kuchar, J. K., "Prototype Conflict Alerting System for Free Flight," AIAA Journal of Guidance, Control, and Dynamics, Vol. 20, No. 4, 1997, pp. 768-773, doi: 10.2514/2.4111.

${ }^{24}$ Contarino, M., "All weather collision avoidance for unmanned aircraft systems," 2010, doi: 10.1117/12.850248.

${ }^{25}$ McCalmont, J., Utt, J., Deenes, M., and Taylor, M., "Sense and avoid technology for Global Hawk and Predator UAVs," 2005.

${ }^{26}$ Graham, S., Chen, W.-Z., De Luca, J., Kay, J., Deschenes, M., Weingarten, N., Raska, V., and Lee, X., "Multiple intruder autonomous avoidance flight test," 2011, AIAA 2011-1420, doi:10.2514/6.2011-1420. 
${ }^{27}$ Cummings, M. and Brzezinski, A., "Global vs. local decision support for multiple independent UAV schedule management," International Journal of Applied Decision Sciences, Vol. 3, No. 3, 2010, pp. 188-205, doi: 10.1504/IJADS.2010.036098.

${ }^{28}$ Ding, J. d., Sprinkle, J. e., Tomlin, C. d., Sastry, S. d. f. g., and Paunicka, J. h., "Reachability calculations for vehicle safety during manned/unmanned vehicle interaction," Journal of Guidance, Control, and Dynamics, Vol. 35, No. 1, 2012, pp. 138-152, doi: 10.2514/1.G000226.

${ }^{29}$ Hurley, R. D., Lind, R., and Kehoe, J. J., "A Torus Based Three Dimensional Motion Planning Model for Very Maneuverable Micro Air Vehicles," AIAA Guidance, Navigation, and Control Conference 2013, AIAA, Boston, MA, 2013, AIAA 2013-4938, doi:10.2514/6.2013-4938.

${ }^{30}$ Huang, S., Feron, E., Reed, G., and Mao, Z.-H., "Compact Configuration of Aircraft Flows at Intersections," IEEE Transactions on Intelligent Transportation Systems, Vol. 15, No. 2, 2014, pp. 771-784, doi: 10.1109/TITS.2013.2287205.

${ }^{31}$ Beard, R. and McLain, T., "Multiple UAV Cooperative Search under Collision Avoidance and Limited Range Communication Constraints," 2003, doi: 10.1109/CDC.2003.1272530.

${ }^{32}$ Liu, W. and Hwang, I., "Probabilistic Aircraft Midair Conflict Resolution Using Stochastic Optimal Control," IEEE Transactions on Intelligent Transportation Systems, Vol. 15, No. 1, 2014, pp. 37-47, doi: 10.1109/TITS.2013.2274999.

${ }^{33}$ Yang, H. I. and Zhao, Y. J., "Trajectory Planning for Autonomous Aerospace Vehicles amid Known Obstacle and Conflicts," AIAA Journal of Guidance, Control, and Dynamics, Vol. 27, No. 6, November-December 2004, pp. 997-1009, doi: $10.2514 / 1.12514$.

${ }^{34}$ Teo, R., Jang, J., and Tomlin, C., "Automated multiple UAV flight - The Stanford DragonFly UAV program," 2004, doi: 10.1109/CDC.2004.1429422.

${ }^{35}$ Richards, A. and How, J., "Decentralized model predictive control of cooperating UAVs," 2004, doi: 10.1109/CDC.2004.1429425.

${ }^{36}$ Mujumdar, A. and Padhi, R., "Reactive Collision Avoidance Using Nonlinear Geometric and Differential Geometric Guidance," AIAA Journal of Guidance, Control, and Dynamics, Vol. 34, No. 1, 2011, pp. 303-311, doi: 10.2514/1.50923.

${ }^{37}$ Chipalkatty, R., Twu, P., Rahmani, A., and Egerstedt, M., "Merging and Spacing of Heterogeneous Aircraft in Support of NextGen," Journal of Guidance, Control, and Dynamics, Vol. 35, No. 5, 2012, pp. 1637-1647, doi:10.2514/1.54076.

${ }^{38}$ Yoo, C., Cho, A., Park, B., Kang, Y., Shim, S., and Lee, I., "ADS-B HILS test for collision avoidance of Smart UAV," 2011.

${ }^{39}$ Ryan, A., Zennaro, M., Howell, A., Sengupta, R., and Hedrick, J., "An overview of emerging results in cooperative UAV control," 2004, doi: 10.1109/CDC.2004.1428700.

${ }^{40}$ Winder, L. F. and Kuchar, J. K., "Evaluation of Collision Avoidance Maneuvers for Parallel Approach," AIAA Journal of Guidance, Control, and Dynamics, Vol. 22, No. 6, November-December 1999, pp. 801-808, doi: 10.2514/2.44818.

${ }^{41}$ Obermeyer, K. J., Oberlin, P., and Darbha, S., "Sampling-Based Path Planning for a Visual Reconnaissance Unmanned Air Vehicle," Journal of Guidance, Control, and Dynamics, Vol. 35, No. 2, 2012, pp. 619-632, doi: 10.2514/1.48949.

${ }^{42}$ Jung, D. and Tsiotras, P., "On-Line Path Generation for Unmanned Aerial Vehicles Using B-Spline Path Templates," Journal of Guidance, Control, and Dynamics, Vol. 36, No. 6, 2013, pp. 1642-1654, doi: 10.2514/1.60780.

${ }^{43}$ Bouabdallah, S. and Siegwart, R., "Full control of a quadrotor," 2007, doi: 10.1109/IROS.2007.4399042.

${ }^{44}$ Beyeler, A., Zufferey, J.-C., and Floreano, D., "Vision-based control of near-obstacle flight," Autonomous Robots, Vol. 27, No. 3, 2009, pp. 201-219, doi: 10.1109/CDC.2002.1184695.

${ }^{45}$ Patel, R. B. and Goulart, P. J., "Trajectory Generation for Aircraft Avoidance Maneuvers Using Online Optimization," AIAA Journal of Guidance, Control, and Dynamics, Vol. 34, No. 1, 2011, pp. 218, doi: 10.2514/1.49518.

${ }^{46}$ Chowdhary, G., Sobers Jr., D. M., Pravitra, C., Christmann, C., Wu, A., Hashimoto, H., Ong, C., Kalghatgi, R., and Johnson, E. N., "Self-Contained Autonomous Indoor Flight with Ranging Sensor Navigation," Journal of Guidance, Control and Dynamic, Vol. 35, No. 6, 2012, pp. 1843-1855, doi:10.2514/1.55410.

${ }^{47}$ Choi, H., Kim, Y., and Hwang, I., "Reactive Collision Avoidance of Unmanned Aerial Vehicles Using aSingle Vision Sensor," Journal of Guidance, Control, and Dynamics, Vol. 36, No. 4, 2013, pp. 1234-1241, doi: 10.2514/1.57131.

${ }^{48}$ Lam, T. M., Mulder, M., van Paassen, M., Mulder, J., and van der Helm, F., "Force-Stiffness Feedback in Uninhabited Aerial Vehicle Teleoperation with Time Delay," AIAA Journal of Guidance, Control, and Dynamics, Vol. 32, No. 3, May-June 2009, pp. 821-836, doi: 10.2514/1.40191.

${ }^{49}$ Muratet, L., Doncieux, S., Briere, Y., and Meyer, J.-A., "A contribution to vision-based autonomous helicopter flight in urban environments," Robotics and Autonomous Systems, Vol. 50, No. 4, 2005, pp. 195-209, doi: 10.1016/j.robot.2004.09.017.

${ }^{50}$ Paielli, R. A., "Modeling Maneuver Dynamics in Air Traffic Conflict Resolution," AIAA Journal of Guidance, Control, and Dynamics, Vol. 26, No. 3, May-June 2003, pp. 407-416, doi: 10.2514/2.5078.

${ }^{51}$ Wang, X., Yadav, V., and Balakrishnan, S., "Cooperative UAV formation flying with obstacle/collision avoidance," IEEE Transactions on Control Systems Technology, Vol. 15, No. 4, 2007, pp. 672-679, doi: 10.1109/TCST.2007.899191.

${ }^{52}$ Prevost, C. G., Desbiens, A., Gagnon, E., and Hodouin, D., "Unamnned Aerial Vehicle Optimal Cooperative Obstacle Avoidance in a Stochastic Dynamic Environment," Journal of Guidance, Control, and Dynamics, Vol. 34, No. 1, 2011, pp. 2944, doi: $10.2514 / 1.50800$.

${ }^{53}$ Klaus, R. and McLain, T., "A Radar-Based, Tree-Branching Sense and Avoid System for Small Unmanned Aircraft," AIAA Guidance, Navigation, and Control Conference 2013, AIAA, Boston, MA, 2013, AIAA 2013-4789, doi:10.2514/6.20134789.

${ }^{54}$ Schmitt, L. and Fichter, W., "Collision-avoidance framework for small fixed-wing unmanned aerial vehicles," Journal of Guidance, Control, and Dynamics, Vol. 37, No. 4, 2014, pp. 1323-1328, doi: 10.2514/1.G000226.

${ }^{55}$ Colozza, A. and Dolce, J., "Initial Feasibility Assessment of a High Altitude Long Endurance Airship," Tech. rep., NASA/CR2003-212724, 2003.

${ }^{56}$ Colozza, A. and Dolce, J., "High-Altitude, Long-Endurance Airships for Coastal Surveillance," Tech. rep., NASA/TM2005-213427, 2005. 
${ }^{57}$ Romeo, G. b., Frulla, G. b., Cestino, E. b., and Corsino, G. b., "HELIPLAT: Design, aerodynamic, structural analysis of long-endurance solar-powered stratospheric platform," Journal of Aircraft, Vol. 41, No. 6, 2004, pp. 1505-1520, doi: $10.2514 / 1.2723$.

${ }^{58}$ Girard, A., Howell, A., and Hedrick, J., "Border patrol and surveillance missions using multiple unmanned air vehicles," Dec 2004, doi: 10.1109/CDC.2004.1428713.

${ }^{59}$ Meester, T., Oomkens, T., and Rudgley, A., "The future is unmanned: Part 4," Maritime by Holland, Vol. 62, No. 6, 2013, pp. 32-43.

${ }^{60}$ Casbeer, D., Beard, R., McLain, T., Li, S.-M., and Mehra, R., "Forest fire monitoring with multiple small UAVs," June 2005, doi: 10.1109/ACC.2005.1470520.

${ }^{61}$ Frew, E., McGee, T., Kim, Z., Xiao, X., Jackson, S., Morimoto, M., Rathinam, S., Padial, J., and Sengupta, R., "Vision-based road-following using a small autonomous aircraft," March 2004, doi: 10.1109/AERO.2004.1368106.

${ }^{62}$ Herwitz, S., Berthold, R., Dunagan, S., Sullivan, D., Fladeland, M., and Brass, J., "UAV Homeland Security Demonstration," AIAA 3rd "Unmanned Unlimited" Technical Conference, Workshop and Exhibit, Infotech@Aerospace Conferences, AIAA, Illinois, MA, 2004, AIAA 2004-6473, doi:10.2514/6.2004-6473. 